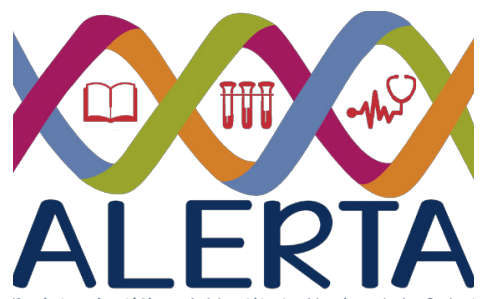

Revista cientifica del Instituto Nacional de Saluo

\title{
Percepción de calidad de vida en usuarios de un programa ambulatorio de diálisis peritoneal
}

Beatriz Elena Archila ${ }^{1}$, Brenda Iveth Beltrán ${ }^{1}$, Marisol Tejada ${ }^{1}$ y Sandra Marcela Arucha ${ }^{1}$

${ }^{1}$ Instituto Tecnológico Escuela Técnica para la Salud
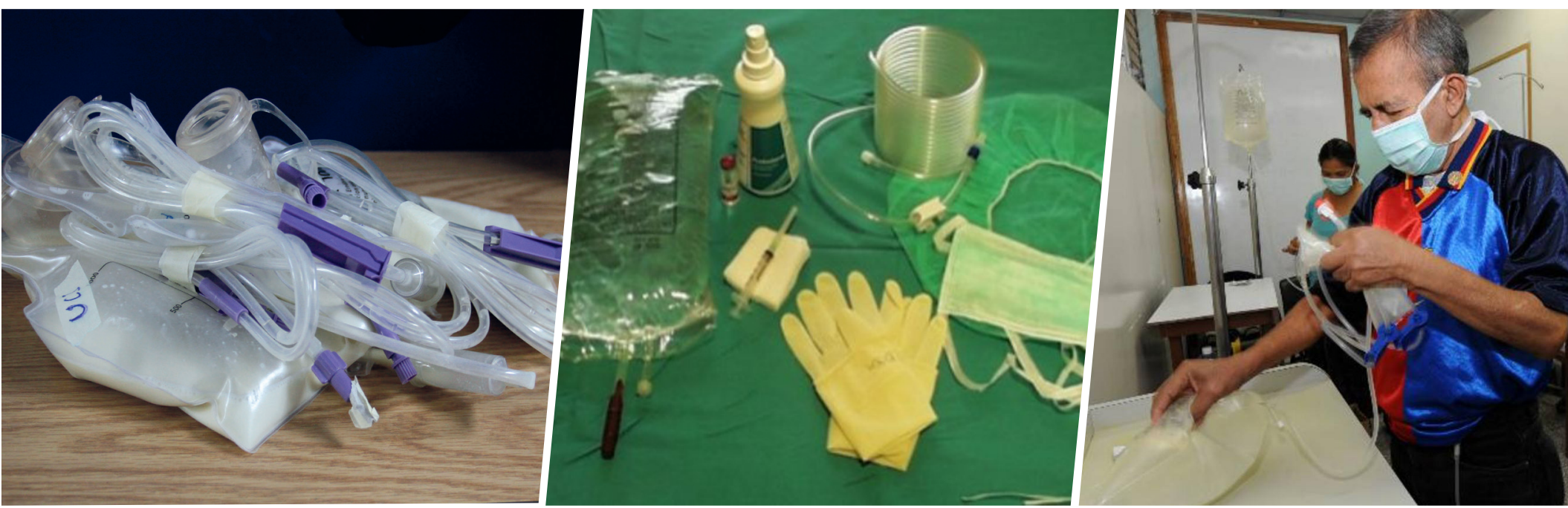

\section{Resumen}

Objetivo. Describir la percepción en la calidad de vida de usuarios del programa ambulatorio de diálisis peritoneal atendidos en Hospital Médico Quirúrgico del Instituto Salvadoreño del Seguro Social. Metodología. Estudio descriptivo transversal. La muestra quedó conformada por 241 personas, participaron 208, distribuidas así: 165 en Diálisis Peritoneal Continua Ambulatoria, 41 en Diálisis Peritoneal Automatizada y 2 cuyos datos fueron perdidos. Se utilizó un cuestionario ad hoc con 41 ítems basados en la escala de tipo Likert. Se aplicaron los tres principios éticos de la Comisión para protección de sujetos humanos en estudios de comportamiento. El procesamiento de la información se realizó en Excel ${ }^{\mathrm{MR}} 2013$ y con el software SPSS versión 22. Resultados. El 97.11\% de usuarios evaluó favorablemente al programa. El 65.86\% expresó una salud física y mental favorable. Conclusiones. Existe una evaluación favorable hacia el programa por sus usuarios, así como una percepción favorable de su salud física y mental.

Palabras claves: Diálisis peritoneal, insuficiencia renal crónica, calidad de vida.

\section{Abstract}

Objective. To describe the perception of quality of life by users of the ambulatory peritoneal dialysis program at Hospital Médico Quirúrgico (third level hospital of Instituto Salvadoreño del Seguro Social). Methodology. Descriptive transversal study. The sample consisted of 241 subjects, of which 208 participated, distributed as follows: 165 in continuous ambulatory peritoneal dialysis, 41 in automated peritoneal dialysis and 2 whose data was lost. An ad hoc questionnaire with 41 items based on Likert scale was used. The three ethical principles of the Commission for the protection of human subjects in behavioral studies, were applied. For reliability and non-parametric tests, the information was analyzed using Excel ${ }^{\mathrm{TM}} 2013$ and SPSS 22. Results. $97.11 \%$ of users evaluated the program favorably. $65.86 \%$ perceived their physical and mental health favorably. Conclusions. There was a favorable evaluation of the program by its users, as well as a favorable perception of their physical and mental health.

Key words: Peritoneal dialysis, chronic renal failure, quality of life. 


\section{Introducción}

La Enfermedad Renal Crónica (ERC) consiste en una destrucción progresiva e irreversible de las nefronas de uno o ambos riñones. En las personas con ERC los estadios se definen según el grado de función renal. Cuando la velocidad de filtración glomerular es inferior a $15 \mathrm{ml} / \mathrm{min}$ ocurre el último estadio (insuficiencia renal crónica o estadio 5). Llegados a esta fase es necesario el tratamiento renal sustitutivo (diálisis o trasplante) ${ }^{1}$.

La ERC se ha convertido en el último cuarto de siglo en una enfermedad de alta prevalencia en la población mundial. La epidemia global de ERC, en todos sus estadios, tiene una prevalencia entre el $10 \%$ y $16 \%$. La prevalencia de Insuficiencia Renal Crónica (IRC) varía entre el $1.4 \%$ y $6.3 \%$ en la población adulta, con una incidencia de diálisis de 285 a 415 pacientes por millón de población (PMP); una prevalencia en diálisis de 1968 a 2288 PMP, con crecimiento anual de un $8 \%$ a $10 \%$, una mortalidad en diálisis de un $20 \%$ y un costo de millones de dólares anuales².

En El Salvador, la IRC constituye un problema de salud pública; el conocimiento de la situación epidemiológica es parcial. Constituye la quinta causa de muerte a nivel nacional en personas mayores de 18 años y la segunda causa de muerte en el sexo masculino. Para el año 2009 la prevalencia de IRC en terapias de reemplazo renal (TRR) fue de $566 \mathrm{PMP}^{3}$.

El estadio final de la ERC es tratable con TRR, entre las cuales se encuentran: la hemodiálisis (HD), diálisis peritoneal (DP) y el trasplante renal (TR $)^{4}$. En El Salvador, durante el año 2014, el Instituto Salvadoreño del Seguro Social (ISSS) atendió a 1742 personas con IRC a través de la $\mathrm{HD}$, diálisis peritoneal continua ambulatoria (DPCA) y ahora la nueva diálisis peritoneal automatizada (DPA) $)^{5}$.
Para agosto del año 2014, el ISSS lanzó el proyecto denominado "Inserción del paciente renal en sustitución a los programas de DPCA y DPA", una estrategia que persigue reducir la saturación de los servicios en el área hospitalaria, reducir los altos costos en la prestación de servicios y una mejora en la calidad de vida de usuarios sometidos a TRR ${ }^{5}$.

Este programa cuenta con diversos indicadores que permiten evaluarlo. Sin embargo, a la fecha no se encuentra un estudio que muestre la evaluación por parte de los usuarios al programa. Este estudio permite un primera evaluación del programa DPCA por parte de los usuarios que son atendidos en el Hospital Médico Quirúrgico del ISSS, utilizando indicadores de estructura, proceso y resultados del programa.

\section{Metodología}

Este es un estudio descriptivo realizado en pacientes con tratamiento de DPCA y DPA, para la evaluación desde la percepción de los usuarios de los programas de tratamiento sustitutivo.

En principio, la población estaba formada por 648 pacientes con IRC distribuidos de la siguiente manera: 449 con DPCA y 199 con DPA. Se realizó el cálculo de la muestra poblacional a través de la fórmula para poblaciones finitas. La muestra poblacional quedó conformada por 241 personas, de las cuales participaron 208 debido a la negación en participar de algunos pacientes seleccionados. Las personas participantes se distribuyeron así: 165 en DPCA, 41 en DPA y dos personas que no sabían cuál era su terapia sustitutiva.

Para la recolección de datos se diseñó una guía de entrevista conformada por 41 ítems. Se evaluaron 
variables relacionadas con el programa DPCA y la percepción de la salud física y mental de los usuarios. Las respuestas a cada ítem fueron categorizadas a través de la escala de Likert. Para la validación del instrumento de recolección de datos, se realizó una prueba piloto con cinco personas que pertenecen al programa DPCA del Hospital Médico Quirúrgico del ISSS mientras esperaban ser atendidas; estas fueron excluidas para participar en la población muestral del estudio. Además, se realizó una validación de contenido del instrumento con expertos en el área de nefrología, dos médicos nefrólogos y dos enfermeras capacitadas en el área.

La recolección de datos fue realizada a través de entrevistas personalizadas dirigidas por los investigadores. Las entrevistas fueron realizadas en las instalaciones del programa DPCA ubicadas en el Hospital Médico Quirúrgico del ISSS mientras los participantes esperaban ser atendidos por el médico, enfermera y nutricionista, como parte de su control mensual. Se realizó lectura del consentimiento informado y se solicitó firma al participante, previo a la aplicación del cuestionario. La duración promedio de cada entrevista fue de diez a quince minutos. Previo a su ejecución, el estudio fue sometido al Comité de Ética del ISSS, obteniendo la aprobación necesaria. La recolección de datos se realizó del 4 al 14 de septiembre del año 2017.

Los datos recolectados fueron digitados a través del software Microsoft Office Excel ${ }^{\text {MR }}$ 2013. Posteriormente se adecuó la base de datos resultante a un formato compatible con el programa Statistical Package for Social Science (SPSS) versión 22, con el cual se administró y analizó la misma.

Para el análisis de los datos se aplicaron distribuciones de frecuencias, medidas de tendencia central y escalas de medición nominal. Además, se obtuvo prueba de confiabilidad alfa de Cronbach para validar las categorías elaboradas con la escala de Likert y obtener la confiabilidad de los datos. Se exploró la salud física y salud mental favorable o desfavorable con base en las siguientes puntuaciones: entre 104 y 78 se clasificó como favorable, una puntuación menor a 52 salud física y mental desfavorable. Los datos obtenido de la evaluación del programa DPCA fueron categorizados con la escala de Likert en cinco alternativas de respuesta: muy de acuerdo, de acuerdo, sin opinión, en desacuerdo y muy en desacuerdo. En este caso se invirtió la ponderación y valor de la escala debido al tipo de enunciado. Una puntuación entre 64 y 48 fue clasificada como una evaluación favorable hacia el programa, si el puntaje era menor a 32, la evaluación se clasificó como desfavorable.

\section{Resultados}

De las personas que participaron, el $66.82 \%$ son hombres y el $33.18 \%$ mujeres. El grupo de edad que presentó la mayor cantidad de pacientes es el que se encuentra entre 49-62 años (36.05\%), el segundo es el de 35-48 años (21.63\%). Las frecuencias para los otros grupos se presentan en el gráfico 1 . Además, se reporta un dato perdido.

En relación al estado civil, el $56.73 \%$ están casados, el $17.30 \%$ están en unión marital, el $16.34 \%$ solteros, el $8.65 \%$ viudos y dos personas divorciadas. Respecto a la ocupación de los participantes, el 41.82\% son empleados, el $29.80 \%$ jubilados, el $24.51 \%$ desempleados y un $3.84 \%$ tienen otro tipo de ocupación. El $44.23 \%$ tiene un nivel académico básico, el $34.13 \%$ un nivel educativo medio, el $16.34 \%$ un nivel de educación superior y el $5.28 \%$ de los participantes no tiene ninguno tipo de estudio. 
Gráfico 1. Distribución de los usuarios del Programa de diálisis peritoneal continua ambulatoria por grupo de edad atendidos en el Instituto Salvadoreño del Seguro Social

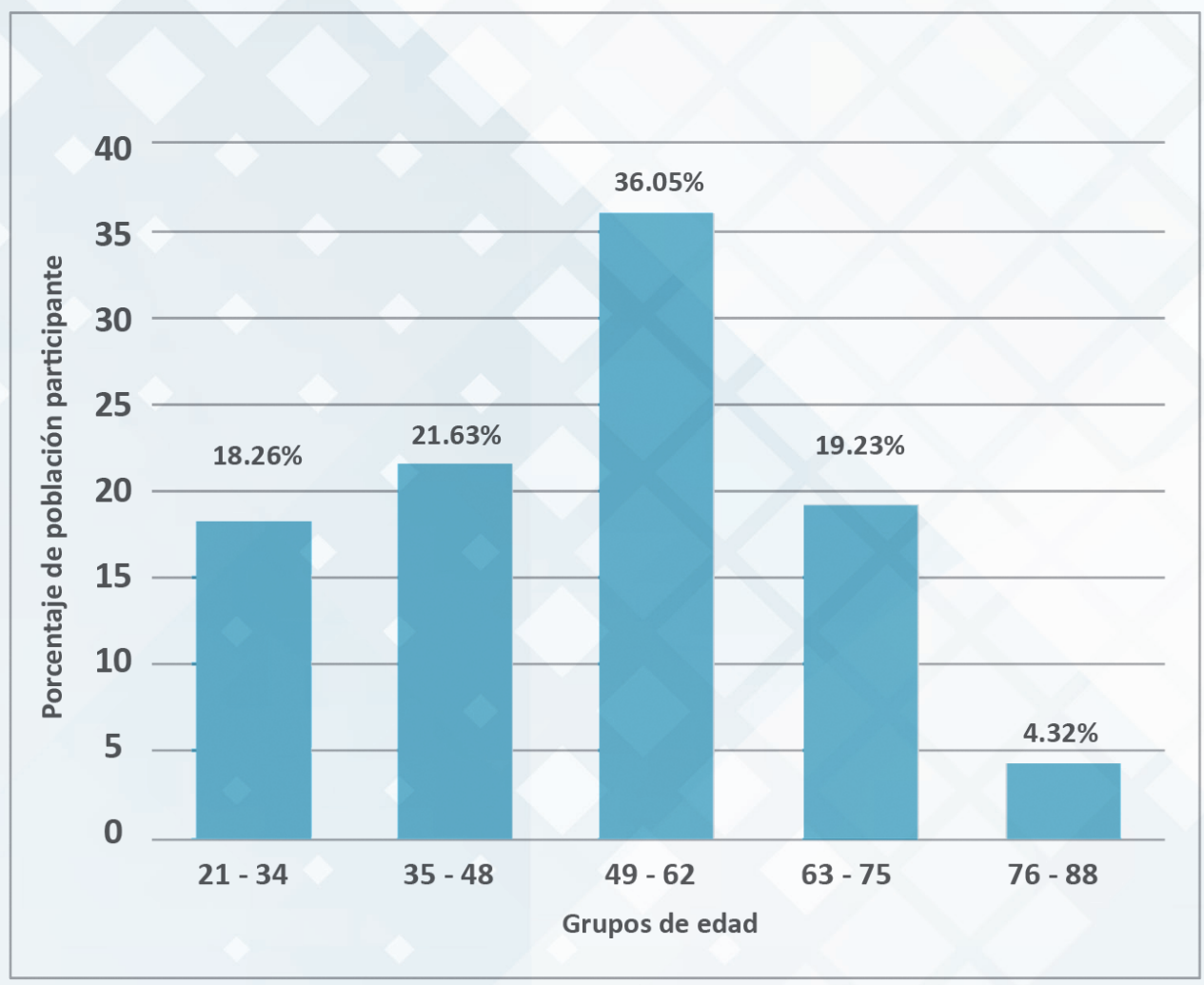

Fuente: Elaboración propia a partir de datos del estudio.

El $58.1 \%$ de los participantes tienen ingresos familiares menores a 300 dólares americanos, el $23.4 \%$ entre 301 y 601 dólares dólares americanos, mientras que un $18.2 \%$ no respondió este ítem. El $50.9 \%$ de los usuarios participa en grupos de apoyo, principalmente en una iglesia, un $2.4 \%$ en otro tipo de grupos, como clubes de adultos mayores o grupos de alcohólicos anónimos y el $45.6 \%$, no participa en ninguno. Al indagar sobre la categorización de los usuarios de acuerdo con la afiliación en el ISSS, el $68.75 \%$ son cotizantes o asegurados, el $29.32 \%$ son beneficiarios de cotizantes y el $1.92 \%$ son usuarios por convenio.

Respecto a la procedencia departamental, el 48.07\% pertenece a San Salvador, el 26.92\% a La Libertad, el $21.15 \%$ a La Paz, el $2.40 \%$ a Cuscatlán y el $1.44 \%$ a
Usulután. La mayoría (78.63\%) son procedentes del área urbana y el $21.35 \%$ del área rural.

En cuanto a la impresión diagnóstica secundaria, el $54.32 \%$ son hipertensos, el $4.32 \%$ diabéticos, el $15.38 \%$ con hipertensos y diabéticos, el $1.92 \%$ diabéticos, el $5.28 \%$ con otra enfermedad y el $15.38 \%$ con ninguna otra enfermedad. Respecto al tiempo de padecer de IRC, el $12.98 \%$ tiene menos de un año, el $45.67 \%$ entre 1 y 5 años, el $26.44 \%$ entre 5 y 10 años, el $13.46 \%$ más de 10 años y una persona no respondió. Del total de pacientes, el $30.76 \%$ ingresó al programa ambulatorio de diálisis peritoneal continua en el año 2016, 19.71\% en el $2015,18.26 \%$ en el $2014,11.5 \%$ en el primer trimestre del 2017 y un $19.69 \%$ entre el 2005 y 2014. 
La mayoría de pacientes (79.32\%) se encontraba en el programa en la modalidad DPCA y un $19.71 \%$ en DPA. Se reportan dos datos perdidos de participantes que no respondieron al ítem.

El $97.11 \%$ de los usuarios expresó estar satisfecho con los logros obtenidos a través del programa. Respecto a la percepción de la salud física y mental de los participantes, el $65.86 \%$ la considera favorable y el $34.14 \%$ desfavorable.

\section{Discusión}

La mayoría de participantes pertenece al sexo masculino, esto es coincidente con datos estadísticos presentados a nivel nacional y mundial, donde el grupo mas prevalente para IRC lo constituye el sexo masculino ${ }^{2,6,11}$.

Las edades de los participantes oscilan entre los 35 y 62 años de edad, en su mayoría. El resto se encuentra entre los 63 y 88 años. Esto pone de manifiesto que la IRC afecta mayormente a la población en edad productiva, acarreando serias implicaciones laborales desde el momento del diagnóstico. Al revisar el nivel ocupacional, cerca de la mitad aún se encuentra laboralmente activo, el resto se encuentra sin empleo; algunos son jubilados o pensionados.

En relación al estado civil, cerca de tres cuartas partes están casados o en unión marital, mientras que el resto está soltero, viudo o divorciado, siendo significativo el apoyo emocional que pueda proporcionar la pareja al paciente con IRC ${ }^{11}$.

En cuanto a si participa o no en grupos de apoyo, la mitad de los participantes lo hace a través de grupos de iglesia y el resto no participa de ningún grupo. Teniendo en cuenta que la situación económica familiar, el apoyo familiar y de red social, se vuelven básicos ante cualquier situación de salud, resulta imprescindible para el caso de los participantes del programa DPCA con IRC, contar con un nivel económico que les permita seguir siendo cabezas de hogar.

Respecto a la procedencia de los participantes por departamentos, San Salvador, La Libertad y La Paz representan la mayor proporción de usuarios comparado con Cuscatlán y Usulután. aproximadamente tres cuartas partes provienen el área urbana y el resto del área rural. Es importante considerar la cobertura de población que proporciona el ISSS, lo cual establece una amplia brecha entre la población atendida por el ISSS y el Minsal2, 6 .

En cuanto a la impresión diagnóstica secundaria, más de la mitad de los participantes con IRC padecen de hipertensión arterial, una mínima parte de diabetes mellitus, mientras que algunos adolecen de ambas enfermedades. De acuerdo con otros estudios ${ }^{1,4}$, se evidencia que los pacientes con diagnóstico reciente padecen de hipertensión, pero la causa más frecuente suele ser la nefropatía diabética, lo cual contrasta con lo encontrado en presente estudio.

Más de tres cuartas partes de los participantes estan en DPCA y el resto en DPA, reportando dos datos perdidos (dos personas no respondieron si se encontraban en DPCA o DPA). La información estadística proporcionada por el departamento de Nefrología del Hospital Médico Quirúrgico del ISSS reporta que para julio de 2016 había inscritos en el programa 449 usuarios en DPCA y 199 en DPA, siendo similares en proporción, a los datos que arrojó el estudio.

Al establecer el tiempo de ingreso al programa DPCA, más de tres cuartas partes dijo estar en él desde el año 2014; sin embargo, menos de la quinta parte refiere haber ingresado antes del 2014. Lo anterior representa un sesgo en la interpretación de la pregunta, por lo que proporcionaron el dato desde que se encuentran en diálisis peritoneal y no de manera específica en DPCA. Haciendo referencia a los datos anteriores, 
respecto al tiempo de ingreso de los participantes al programa, resulta importante señalar un registro nacional de España ${ }^{7,8}$ que muestra la evolución de la mortalidad y consistentemente permite apreciar que la DP domiciliaria supera siempre en supervivencia a la $\mathrm{HD}^{7,8}$. Los pacientes en DP tienen mayor supervivencia, no sólo al inicio del tratamiento, sino también mantenida a los 46 meses de seguimiento ${ }^{7,8}$. Esta situación se mantiene en todos los subgrupos analizados, mayores y menores de 65 años, hombres y mujeres, diabéticos y no diabéticos. Al confrontar la información obtenida con el referente citado, la mayoría de usuarios desde que ingresaron al programa tiene una sobrevivencia de más de 36 meses, lo cual suele ser consistente con los datos registrados por España ${ }^{7,8}$.

En su mayoría, la evaluación realizada por los participantes del programa DPCA es favorable. Respecto a la percepción de salud física y mental, más de la mitad de los participantes la percibe como favorable, es decir, a pesar de sus limitaciones de salud física, aún logran cumplir sus diferentes roles en el ámbito laboral y social. Mientras que para el resto, es desfavorable, asumiendo el concepto de calidad de vida como un estado de salud funcional, percepción de buena salud, satisfacción con la vida y habilidad para competir de los participantes ${ }^{10}$.

Tal como se planteó inicialmente no existen estudios previos a nivel nacional ${ }^{11,12}$ e internacional ${ }^{7,8,9}$ que permitan determinar la percepción del programa DPCA $^{13,14}$ y la calidad de vida de los participantes.

Este estudio debe profundizarse realizando análisis con instrumentos estandarizados para medir la calidad de vida. Además, se deben realizar evaluaciones de impacto de los programas como parte de una mejora continua.

\section{Conclusiones}

Se plantea una evaluación favorable de los usuarios hacia el programa y una calidad de vida favorable en la mayoría de los participantes. A pesar que el modelo utilizado no persigue demostrar la asociación entre variables, resulta muy valioso señalar la percepción favorable que los participantes expresan sobre el programa DPCA. Finalmente, el estudio pone de manifiesto la percepción de la calidad de vida actual de los usuarios: para la mayoría de participantes es favorable.

\section{Conflicto de intereses}

Los autores declaran no tener conflicto de intereses en aquellas actividades que pudieran introducir sesgos en los resultados del trabajo.

\section{Financiación}

El presente estudio ha sido financiado por el Instituto Tecnológico Escuela Técnica para la Salud. 


\section{Referencias bibliográficas}

1. Lewis SM, Heitkemper M, Dirksen SR. Enfermería Medico quirúrgica: Valoración y Cuidados de Problemas Clínicos. Madrid: Elsevier; 2004. P. 1-5172.

2. Instituto Nacional de Salud. Investigación en salud renal. [en línea]. El Salvador: Ministerio de Salud. [Consultado el 13 octubre 2016]. Disponible en: http://ins.salud.gob.sv/index.php/temas/ investigacion/investigacionensaludrenal\#introducci\%C3\%B3n

3. Cruz A, de María A, Leiva I, Ayala N, Hernández C. Guías clínicas de atención a las personas con enfermedad renal crónica [Internet]. San Salvador: Instituto Salvadoreño de Bienestar Magisterial; 2013 [Citado 13 octubre 2016]. p. 1-31. Disponible en: http:// www.isbm.gob.sv/phocadownload/guias_medicas/GUIA\%20 CLINICAS\%20ENFERMEDAD\%20RENAL\%20CRONICA $\% 20$ ERC,\%2004102013.pdf

4. Chacón JE, Guzmán KS. Proyecto de mejoramiento de la calidad en el proceso de diálisis peritoneal continua ambulatoria. Instituto Salvadoreño del Seguro Social. San Salvador; 2014.

5. Noticias Ciudadano. ISSS anuncia implementación de Diálisis Peritoneal Automatizada [Internet]. San Salvador: Instituto Salvadoreño del Seguro Social de El Salvador; 2015. [Citado 13 octubre 2016]. Disponible en: http://www.isss.gob.sv/\%20index. php? option $=$ com_content $\&$ view $=$ article $\&$ catid $=1$ :noti\%20ciasciudadano\&id=1378:-isss-anuncia-implementacion-dedialisisperitoneal-automatizada\&Itemid $=77$

6. Día Mundial del Riñón. Situación de la enfermedad renal crónica en El Salvador [Internet]. San Salvador: Organización Panamericana para la Salud/Organización Mundial de la Salud; 2017 [Citado 13 marzo 2017]. p. 1-2. Disponible en: http://www.paho.org/els/ index.php?option =com_docman\&view = download\&alias $=1196$ situacion-renal-els \&category_slug = materiales-decampanas\&Itemid $=364$

7. Arrieta J, Rodríguez-Carmona A, Remón C, Pérez-Fontan $M$, Ortega R, Sánchez Tomero JA, Selgas R. La diálisis peritoneal es la mejor alternativa coste-efectiva para la sostenibilidad del tratamiento con diálisis. Revista Nefrología [Internet]. 2011 [Citado 5 de mayo de 2016]; 31(5): p. 505-13. Disponible en: http://scielo. isciii.es/pdf/nefrologia/v31n5/editorial.pdf

8. de Francisco ALM. Sostenibilidad y equidad del tratamiento sustitutivo de la función renal en España. Revista Nefrología [Internet]. 2011 [Citado 5 de mayo de 2016]; 31(3): p. 241-6.
Disponible en: http://www.revistanefrologia.com/es-publicacionnefroplus-articulo-sostenibilidad-equidad-del-tratamientosustitutivo-funcion-renal-espana-X0211699511052071

9. Sandoval-Jurado L, Ceballos-Martínez ZI, Navarrete-Novelo C, González-Hernández F, Hernández-Colín V. Calidad de vida en pacientes con diálisis peritoneal continua ambulatoria. Rev Med Inst Mex Seguro Soc [Internet]. 2007 [Citado 3 de marzo de 2017]; 45(2): p. 105-109. Disponible en: http://www.redalyc.org/articulo. oa?id=457745526002

10. Misrachi C, Gaete J, Espinoza I. Calidad de vida y salud, instrumentos de medición. Mag int coll dent 2002;10(1): p.94-107.

11. Álvarez WM, González JD. Calidad de vida de los pacientes con insuficiencia renal crónica tratados con diálisis peritoneal intermitente automatizada y diálisis peritoneal continua ambulatoria en el Hospital Nacional Regional San Juan de Dios de la ciudad de San Miguel. Período de enero a junio de 2010 [tesis doctoral]. [San Miguel, El Salvador]: Universidad de El Salvador, Facultad Multidisciplinaria Oriental; 2010.

12. Arévalo Henríquez, FA. Calidad de vida en pacientes con insuficiencia renal crónica en tratamiento con diálisis peritoneal continua ambulatoria, en el HNSJDSM durante el período de enero 2013 a diciembre 2015. En: III Congreso Nacional de Investigaciones en Salud: 2 de diciembre 2016, San Salvador, El Salvador. INS; 2016.

13. Naranjo M. Evaluación de programas de salud. Comunidad y Salud [Internet]. 2006 [Citado 25 de octubre de 2016]; 4(2): p. 3437. Disponible en: http://www.scielo.org.ve/scielo.php?script=sci_ arttext\&pid=S1690-32932006000200005

14. Scoppetta O. Discusión sobre la evaluación de impacto de programas y proyectos sociales en salud pública. Univ. Psychol [Internet]. 2006 [Citado 27 de octubre de 2016]; 5(3): p. 695-703. Disponible en: http://www.scielo.org.co/pdf/rups/v5n3/v5n3a21.pdf

\section{Forma recomendada de citar}

Archila B, Beltrán BI, Tejada M, Arucha SM. Percepción de calidad de vida en usuarios de un programa ambulatorio de diálisis peritoneal. Revista ALERTA. 2018;1(1):35-41. 